\title{
Breakdown of Mineral Oil: Effect of Electrode Geometry and Rate of Voltage Rise
}

\author{
Mark P. Wilson, Igor V. Timoshkin, Martin J. Given, Scott J. MacGregor, T. Wang \\ Institute for Energy and Environment, Dept. Electronic \& Electrical Engineering, University of Strathclyde \\ Royal College Building, 204 George Street \\ Glasgow, G1 1XW, UK
}

\author{
Mark A. Sinclair, Ken J. Thomas \\ Pulsed Power Group \\ AWE Aldermaston \\ Reading, RG7 4PR, UK \\ and Jane M. Lehr \\ Sandia National Laboratories \\ P.O. Box 5800, MS 1193 \\ Albuquerque, NM 87185-1193, USA
}

\begin{abstract}
Experimental data on the propagation of streamers in mineral oil is important for the design of high-voltage systems in the power and pulsed-power industries. In the present study, breakdown voltages and pre-breakdown delay times were measured for planeparallel electrodes, and for two non-uniform electrode arrangements. For each geometry, the breakdown characteristics were determined for impulses of rise-time $100 \mathrm{~ns}$, and also rise-time $1 \mu \mathrm{s}$. The maximum rate of voltage rise $(d V / d t)$ was $4 \mathrm{MV} / \mu \mathrm{s}$. For the non-uniform geometries with inter-electrode gap length of $8.5 \mathrm{~mm}$, the time to breakdown was 2.5-3 times longer for impulses of rise-time $1 \mu$ s than for $100 \mathrm{~ns}$ risetime. The time-to-breakdown data suggest that streamer propagation velocity increases with higher values of $d V / d t$. For example, the estimated propagation velocity for pinplane geometry with a $1 \mu$ s rise-time is $10-12 \mathrm{~km} / \mathrm{s}$. At $100 \mathrm{~ns}$ rise-time for the same electrode geometry, the average propagation velocity exceeds $40 \mathrm{~km} / \mathrm{s}$. The results are compared with data previously generated in parallel liquid-solid gaps, and it is concluded that the time to breakdown is longer, and that higher applied fields are required to initiate breakdown, in open oil gaps compared to the case when a solid spacer is present. The results presented are intended to provide reference data for designers of oil-immersed, high-voltage systems such as power transformers and pulsed-power supplies.
\end{abstract}

Index Terms - Breakdown voltage, dielectric breakdown, dielectric liquids, oil insulation, power transformers, pulsed power supplies.

\section{INTRODUCTION}

EXPERIMENTAL data on the breakdown of mineral oil under different conditions of applied voltage is important for the design of high-voltage systems in the power and pulsed-power industries.

Mineral oil is used as an insulating liquid in pulsed-power systems such as oil-immersed Marx generators. The dielectric properties of mineral oil, including its breakdown characteristics in different problematic electrode topologies, are important for the design and development of pulsed-power systems and their elements, including pulsed transmission lines and plasma closing switches $[1,2]$. In such systems, the insulating liquid can be stressed with high impulsive electric fields of magnitude up to several $\mathrm{MV} / \mathrm{cm}$ and duration up to hundreds of microseconds. The present work is focused on investigation of the effect of electrode geometry and rate of voltage rise on the breakdown characteristics of mineral oil. Three different electrode topologies were used: pin-plane and collar-plane topologies which generate highly non-uniform electric field; and plane-parallel topology, used as a reference electrode arrangement. These electrode configurations represent typical problematic topologies found in large-scale 
pulsed-power systems employed by AWE and Sandia National Laboratories, and it is necessary to examine and understand the dielectric behavior of such electrode systems to enable the reliable operation of high-voltage machines.

The present study formed part of a larger investigation to determine the voltages and pre-breakdown delay times associated with breakdown of different polymer surfaces immersed in mineral oil [3-7]. Described herein are the differences in breakdown voltage, time to breakdown, and implied average streamer propagation velocity in open oil gaps without a solid spacer, for the electrode geometries and voltage rise-times used for surface breakdown measurements in [3-6].

Breakdown voltages and pre-breakdown delay times were measured for plane-parallel electrodes, and for two types of nonuniform arrangement when the oil gap was subjected to overvoltages. For each geometry, the breakdown characteristics were determined for impulses of rise-time $100 \mathrm{~ns}$, and also rise-time $1 \mu \mathrm{s}$. The maximum applied voltage level was $400 \mathrm{kV}$, giving a maximum rate of voltage rise $(d V / d t)$ of $4 \mathrm{MV} / \mu \mathrm{s}$. In a second set of measurements, termed breakdown-initiation measurements, impulses of increasing peak voltage were applied to oil gaps until breakdown events occurred.

A polarity effect on breakdown was observed in [8] when transformer oil was tested in a point-plane geometry. The breakdown strength was found to be $\sim 50 \%$ higher for a negative point than a positive point at short breakdown times (1-2.5 ns). The polarity effect has been explained in [9-12] by an effective reduction in the inter-electrode gap length under positive polarity, as electrons are generated in the liquid and attracted to the positive-point electrode, and the conducting channel formed as a result of ionization of the liquid between the anode and the electron cloud acts as an extension of the needle anode. In the negative impulse regime, a negative volumetric space charge is formed in front of the needle, reducing the field gradient and impeding the injection of further electrons, meaning that higher applied voltages are required to cause breakdown. Impulse voltages of negative polarity were therefore used throughout the present study, corresponding with the polarity used in [3-6].

Both wave-shapes $(100 / 600 \mathrm{~ns}$ and $1 / 6.5 \mu \mathrm{s})$ utilized in the study will be of interest to designers of pulsed-power supplies and components. The $1 / 6.5 \mu$ s wave-shape used is similar to a short-tail lightning impulse, meaning that the results may also be of interest to designers of oil-immersed, high-voltage systems such as power transformers.

\section{EXPERIMENTAL ARRANGEMENT}

The experimental system comprised a high-voltage impulse generator, test cell, and voltage monitoring diagnostics. The impulse generator is a ten-stage, air-insulated, inverting Marx generator, capable of producing output voltages in the region of $500 \mathrm{kV}$. The erected capacitance of the Marx is $8 \mathrm{nF}$, and switching is achieved by the depressurization of an in-line spark-column, consisting of ten discrete spark-gap switches, filled with dry air. The storage capacitors are charged by a positive-polarity, 100-kV, Glassman high-voltage dc supply.

The values of the wave-shaping resistors were varied in order to achieve different voltage rise- and fall-times. To generate impulses of rise-time $100 \mathrm{~ns}$ (600 ns to half-peak value), a $600 \Omega$ aqueous copper sulphate $\left(\mathrm{CuSO}_{4}\right)$ wave-front resistor was utilized along with a $200 \Omega \mathrm{CuSO}_{4}$ wave-tail resistor. In a second configuration, an $800 \Omega \mathrm{CuSO}_{4}$ wave-tail resistor and a $1.6 \mathrm{k} \Omega \mathrm{CuSO}_{4}$ wave-front resistor were used to achieve a voltage rise-time of $\sim 1 \mu \mathrm{s}$ ( $6.5 \mu$ s to half-peak value); in this configuration, a wave-front capacitance of $200 \mathrm{pF}$ was also connected in parallel with the test-cell load.

The test cell consists of a pair of 50-mm-diameter aluminium electrodes, immersed in $20 \mathrm{~L}$ of EOS Ltd. L10B reclaimed insulating oil. The insulating oil received from EOS Ltd. was stored in plastic barrels under atmospheric conditions; the water content and purity of this oil were not examined. These conditions are typical for the practical usage of mineral oils in the pulsed-power industry, and therefore it is important to know the dielectric characteristics of this oil "as received" from the supplier.

Three different electrode arrangements were tested, with the earthed electrode consisting of a 50-mm-diameter plane in all three geometries. Three different high-voltage electrodes were utilized, as illustrated in Figure 1. Both the type A and type B electrodes were designed to initiate discharges in close proximity to the surface of cylindrical solids of diameter $25 \mathrm{~mm}$ [3-6]. The first type of electrode, type A, had a 3-mmlong, 1-mm-diameter, tungsten-alloy pin protruding from the main body to provide a point-plane electrode geometry. The second type, type B, also provided non-uniform field geometry, with a 25-mm-diameter aluminum collar centered on the electrode body. The collar was machined at an angle of $45^{\circ}$, and the diameter at the tip was again $1 \mathrm{~mm}$. For the voltage levels (up to $400 \mathrm{kV}$ ) and inter-electrode gap lengths (minimum of $8.5 \mathrm{~mm}$ ) studied herein, the estimated peak electric field levels at the tips of the type A and type B electrodes are of the order of 1-4 MV/cm. The third type of high-voltage electrode, type $\mathrm{C}$, was a 50-mm-diameter plane with no modifications, providing plane-parallel electrode geometry.

The impulse voltage wave-forms were monitored using a Tektronix P6015A high-voltage probe connected to a tap-off point on a Samtech Ltd. DE(LRP)-02 $\mathrm{CuSO}_{4}$ voltage divider (10 $\mathrm{k} \Omega$ input impedance), giving a measurement system with an overall division ratio of 10,000:1. The voltage wave-forms were viewed and recorded on a Tektronix TDS3032 digital storage oscilloscope; typical wave-forms corresponding to those used in the measurements reported here, but associated with surface breakdown events, can be found in [3-6].

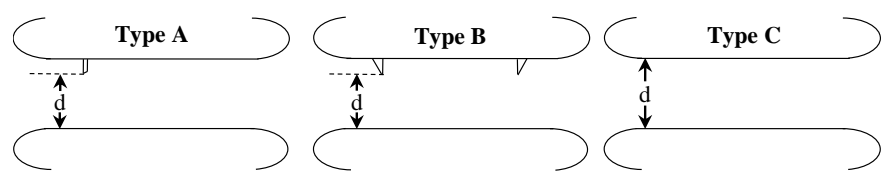

Figure 1. Sketch of the different electrode arrangements used throughout the study. The inter-electrode gap length, $d$, is shown for each geometry. 


\section{OVER-VOLTAGE TESTS}

Oil gaps of each of the geometries shown in Figure 1 were subjected to impulse voltages of negative polarity. For each geometry, five impulses of rise-time $100 \mathrm{~ns}$ were first applied across the electrodes. The peak voltage applied to the gap was fixed at $-400 \mathrm{kV}$ for these initial measurements, resulting in breakdown of the oil on the falling edge of the impulse. The voltage at breakdown, $V_{b r}$, and the pre-breakdown delay time, $t_{b r}$, was recorded for each breakdown event (see Figure 3).

The voltage rise-time was then increased by an order of magnitude to $1 \mu \mathrm{s}$ in order to study pre-breakdown delay times associated with breakdown events occurring either on the impulse rising edge or around the peak of the impulse, rather than on the impulse falling edge. A peak voltage of $-350 \mathrm{kV}$ was applied across the gap five times, again, for each electrode geometry. The peak voltage magnitude was limited to $350 \mathrm{kV}$ in this configuration due to the voltage rating of the 200-pF wave-front capacitor.

\subsection{NON-UNIFORM FIELDS}

The values of the voltage at breakdown for the pin and collar high-voltage electrodes $(d=8.5 \mathrm{~mm})$ are listed in Table 1. It is clear that for the 100-ns rise-time, the voltage at breakdown is higher for the pin electrode (average $V_{b r}$ $385 \pm 11 \mathrm{kV}$ ) than for the collar electrode (average $V_{b r}$ $307 \pm 14 \mathrm{kV})$. At $1 \mu \mathrm{s}$ rise-time, the voltage at breakdown is very similar for both the pin-plane (average $V_{b r} 335 \pm 2 \mathrm{kV}$ ) and the collar-plane (average $V_{b r} 336 \pm 1 \mathrm{kV}$ ) oil gap, and there is only a small variation in the voltage at breakdown over the five shots for either type of gap. The \pm values quoted throughout this paper correspond to the standard deviation.

Displayed in Figure 2 are time-to-breakdown data for both non-uniform geometries $(d=8.5 \mathrm{~mm})$ at $100 \mathrm{~ns}$ rise-time. It is clear that the times to breakdown are much longer for the collar electrode, and in fact the average pre-breakdown delay time of $499 \pm 43 \mathrm{~ns}$ over the five shots is over twice that of the average time for the pin electrode $(223 \pm 15 \mathrm{~ns})$.

It is important to note that although the voltage at breakdown is lower for the collar electrode at $100 \mathrm{~ns}$ rise-time, the time to breakdown, as shown in Figure 2, is significantly longer. As breakdown occurred on the falling edge of the impulse, the reduction in the measured voltage at breakdown for the collar electrode is a result of the longer time to breakdown, as illustrated in Figure 3.

Table 1. Voltage at Breakdown, $V_{b r}$, for Non-uniform Field Geometries (5 Individual Breakdowns).

\begin{tabular}{c|c|c|c||c|c}
\hline \multicolumn{2}{c|}{ Rise-time } & \multicolumn{2}{c||}{$100 \mathrm{~ns}$} & \multicolumn{2}{c}{$1 \mu \mathrm{s}$} \\
\hline $\begin{array}{c}\text { High-voltage } \\
\text { electrode }\end{array}$ & Pin & Collar & Pin & Collar \\
\hline \multirow{4}{*}{$V_{b r}(\mathrm{kV})$} & 1 & 378 & 318 & 336 & 336 \\
\cline { 2 - 6 } & 2 & 394 & 320 & 336 & 336 \\
\cline { 2 - 6 } & 3 & 378 & 300 & 338 & 336 \\
\cline { 2 - 6 } & 4 & 374 & 286 & 334 & 336 \\
\cline { 2 - 6 } & 5 & 400 & 310 & 332 & 334 \\
\hline
\end{tabular}

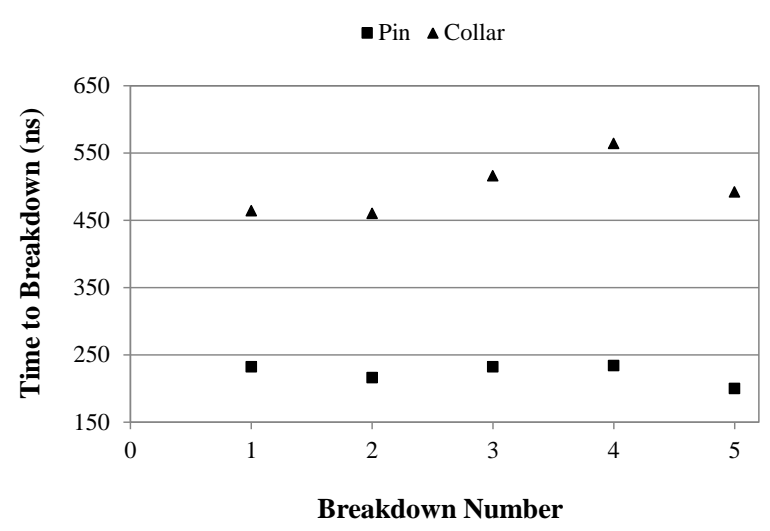

Figure 2. Time to breakdown versus breakdown number (5 individual breakdowns) for non-uniform geometries for rise-time $100 \mathrm{~ns}$.

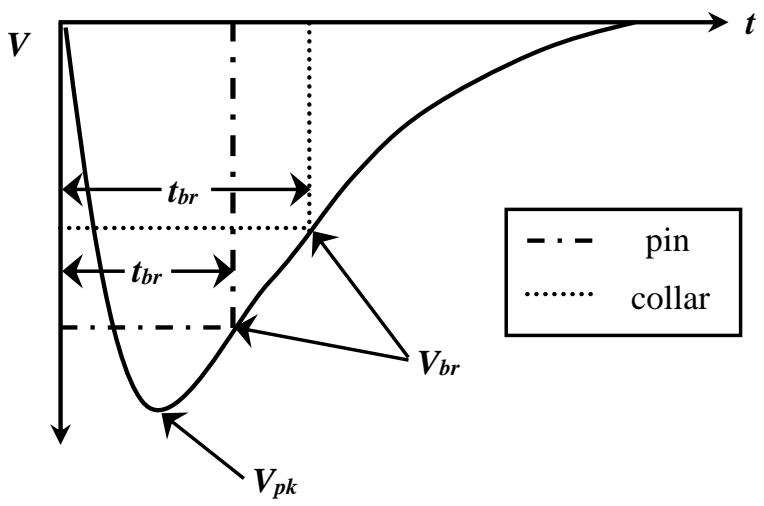

Figure 3. Sketch illustrating the differences in time to breakdown, $t_{b r}$, and hence voltage at breakdown, $V_{b r}$, for impulses of rise-time $100 \mathrm{~ns}$. Also indicated is the peak applied voltage, $V_{p k}$.

Although the voltage at breakdown for the pin and collar electrodes is very similar for rise-time $1 \mu \mathrm{s}$, the pre-breakdown delay times were again longer for the collar electrode. Figure 4 provides a comparison of the breakdown-time data for $1 \mu \mathrm{s}$ rise-time $(d=8.5 \mathrm{~mm})$. While breakdown events occurred on the rising edge for the pin-plane geometry, it is clear that times to breakdown longer than $1 \mu \mathrm{s}$ were obtained for collar-plane geometry. As the corresponding breakdown voltage was always within $5 \%$ of the peak voltage, the data were accepted in order to provide a comparison with the pin-plane geometry. The average breakdown times are $766 \pm 45 \mathrm{~ns}$ and $1260 \pm 89 \mathrm{~ns}$ for the pin-plane and the collar-plane oil gaps, respectively. This behaviour is discussed by way of analysis of the peak electrical field for the pin and collar electrodes in Section 5.

When the data are compared for both rise-times for pinplane electrode geometry, the time to breakdown is approximately $500 \mathrm{~ns}$ shorter for $100 \mathrm{~ns}$ rise-time impulses than for impulses of $1 \mu$ s rise-time. For the collar high-voltage electrode, again the $100 \mathrm{~ns}$ rise-time impulses provide a shorter time to breakdown, this time the difference compared to the $1 \mu \mathrm{s}$ impulses is around $700 \mathrm{~ns}$. 


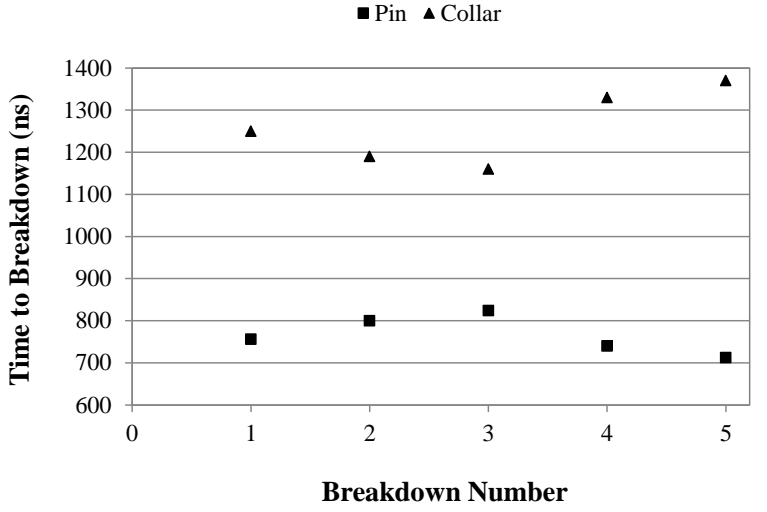

Figure 4. Time to breakdown versus breakdown number (5 individual breakdowns) for non-uniform geometries for rise-time $1 \mu$ s.

At such short time intervals and high $d V / d t$, a streamer mechanism can be assumed to be integral to the breakdown process. Lesaint [13] states that "the term "streamer" remains widely used in liquids to designate all propagating prebreakdown phenomena", and it should be noted that the same approach has been taken here. While the actual velocity of the streamer that propagates to the point of breakdown cannot be accurately determined without spatial and temporal imaging of the streamer development, an average streamer velocity, estimated by dividing the inter-electrode gap length by the time to breakdown, can provide a minimum implied velocity of streamer propagation. It is recognized that the propagation velocity will not be constant over the full interelectrode gap length. Torshin [14] discusses the propagation of leader discharges, more commonly discussed in relation to electrical breakdown of air, in mineral oil. Leaders can close the inter-electrode gap, travelling with a higher velocity than in the earlier discharge propagation phases.

The implied average streamer velocities can provide a basis for comparison with the results from other studies, and these are listed in Table 2 for the various non-uniform field tests. Based upon the classification of streamer propagation modes discussed elsewhere [15-18], the data suggest that faster streamer velocities are found under higher $d V / d t$. For example, the propagation velocity for pin-plane geometry with a $1 \mu \mathrm{s}$ rise-time is $10-12 \mathrm{~km} / \mathrm{s}$. At $100 \mathrm{~ns}$ rise-time for the same electrode geometry, the average propagation velocity exceeds $40 \mathrm{~km} / \mathrm{s}$ for the final breakdown.

Table 2. Implied Average Streamer Propagation Velocities Under Nonuniform Field Geometries (5 Individual Breakdowns).

\begin{tabular}{c|c|c|c|c|c}
\hline \multirow{2}{*}{ Electrodes (rise-time) } & \multicolumn{5}{|c}{ Average Streamer } \\
\cline { 2 - 6 } & 1 & 2 & 3 & 4 & 5 \\
\hline Pin-plane $(100 \mathrm{~ns})$ & 37 & 39 & 37 & 36 & 43 \\
\hline Collar-plane $(100 \mathrm{~ns})$ & 18 & 19 & 17 & 15 & 17 \\
\hline \hline Pin-plane $(1 \mu \mathrm{s})$ & 11 & 11 & 10 & 12 & 12 \\
\hline Collar-plane $(1 \mu \mathrm{s})$ & 7 & 7 & 7 & 6 & 6 \\
\hline
\end{tabular}

Badent et al [16] studied negative streamers in insulating oil and, according to their classification, secondary streamers have average velocity up to $40 \mathrm{~km} / \mathrm{s}$, and tertiary streamers have velocity exceeding $47 \mathrm{~km} / \mathrm{s}$. Massala and Lesaint [17] define fast streamers in mineral oil as having a velocity higher than $50 \mathrm{~km} / \mathrm{s}$. Duy et al [18] classify streamers simply as slow for average velocity $1.5-3 \mathrm{~km} / \mathrm{s}$, and fast for average velocity $20-200 \mathrm{~km} / \mathrm{s}$, although this classification is in relation to the propagation of positive streamers.

\subsection{UNIFORM FIELDS}

The voltages at breakdown, $V_{b r}$, recorded for the different rise-times for plane-parallel geometry are listed in Table 3 along with the times to breakdown, $t_{b r}$. It must be noted that while the inter-electrode gap length was $8 \mathrm{~mm}$ for $100 \mathrm{~ns}$ risetime, it was necessary to reduce the gap length to $4 \mathrm{~mm}$ for $1 \mu \mathrm{s}$ rise-time in order to ensure that breakdown events occurred only on the rising edge of the applied impulse. When an 8-mm gap length was tested under the longer rise-time regime, breakdown events commonly occurred up to several $\mu \mathrm{s}$ after the impulse peak. The main purpose of the results is to provide reference data for comparison with the breakdown characteristics of the various liquid-solid gaps tested in [3-6], and the gap lengths tested herein correspond to the lengths of the solid surfaces subjected to the relevant impulse wave-shape in these papers. The time to breakdown with and without solid spacers is compared and discussed in Section 5.

Table 3. Voltage at Breakdown, $V_{b r}$, and Time to Breakdown, $t_{b r}$, for Overvoltage Measurements with Plane-parallel Electrodes.

(5 Individual Breakdowns).

\begin{tabular}{c|c|c|c}
\hline $\begin{array}{c}\text { Rise-time } \\
\text { (inter-electrode gap) }\end{array}$ & & $V_{b r}(\mathrm{kV})$ & $t b r(\mathrm{~ns})$ \\
\hline \multirow{4}{*}{$\begin{array}{c}100 \mathrm{~ns} \\
(d=8 \mathrm{~mm})\end{array}$} & 1 & 350 & 378 \\
\cline { 2 - 4 } & 2 & 340 & 350 \\
\cline { 2 - 4 } & 3 & 356 & 312 \\
\cline { 2 - 4 } & 4 & 336 & 376 \\
\cline { 2 - 4 } & 5 & 332 & 364 \\
\hline \hline \multirow{4}{*}{$\begin{array}{c}1 \mu \mathrm{s} \\
(d=4 \mathrm{~mm})\end{array}$} & 1 & 284 & 416 \\
\cline { 2 - 4 } & 2 & 306 & 480 \\
\cline { 2 - 4 } & 4 & 296 & 472 \\
\cline { 2 - 4 } & 5 & 290 & 448 \\
\hline
\end{tabular}

Table 4. Implied Average Streamer Propagation Velocities with Planeparallel Electrodes (5 Individual Breakdowns).

\begin{tabular}{c|c|c|c|c|c}
\hline \multirow{2}{*}{ Electrodes (rise-time) } & \multicolumn{5}{|c}{ Average Streamer } \\
\cline { 2 - 6 } & 1 & 2 & 3 & 4 & 5 \\
\hline Plane-parallel $(100 \mathrm{~ns})$ & 21 & 23 & 26 & 21 & 22 \\
\hline \hline Plane-parallel $(1 \mu \mathrm{s})$ & 10 & 8 & 9 & 9 & 10 \\
\hline
\end{tabular}

Forster and Wong [19] have previously reported on streamers in hydrocarbons under uniform-field conditions similar to those reported herein. The average electric field magnitude in [19] was $300-500 \mathrm{kV} / \mathrm{cm}$, compared to 500 $875 \mathrm{kV} / \mathrm{cm}$ in this paper. The implied average streamer propagation velocities for plane-parallel electrode geometry 
for both impulse wave-shapes applied in the present study are displayed in Table 4.

\section{BREAKDOWN-INITIATION TESTS}

Using the 100-ns rise-time, oil gaps were then subjected to a test in order to determine the peak applied voltages required to initiate breakdown. This involved subjecting the oil gap to multiple negative-polarity impulses, the majority of which did not result in breakdown, with the following test sequence. For both non-uniform geometries, where the electrode separation, $d$, was $10 \mathrm{~mm}$, a peak voltage of $-200 \mathrm{kV}$ (average applied field $\left(V_{p k} / d\right)$ of $\left.200 \mathrm{kV} / \mathrm{cm}\right)$ was initially applied across the electrodes. For uniform plane-parallel geometry, where the electrode separation was $4 \mathrm{~mm}$, the voltage initially applied was $-120 \mathrm{kV}$ (average applied field $300 \mathrm{kV} / \mathrm{cm}$ ). No breakdown events were observed at these levels of applied voltage. For each electrode geometry, the charging voltage was raised in steps of $2 \mathrm{kV}(\sim 20 \mathrm{kV}$ increase in output voltage) until a breakdown occurred, upon which the peak applied voltage, the voltage at breakdown, and the delay time to breakdown were recorded (see Figure 3 ). The charging voltage was then reduced to return the peak applied voltage to $-200 \mathrm{kV}$ or $-120 \mathrm{kV}$, depending upon the electrode configuration, and the process was repeated until a total of five breakdowns had occurred. The time between impulse voltage applications was five minutes.

When breakdown-initiation measurements were attempted for the longer rise-time of $1 \mu \mathrm{s}$, which was utilized to study rising-edge breakdown events in Section 3, breakdown events generally occurred on the falling edge, with pre-breakdown delay times up to $4 \mu \mathrm{s}$, and this type of measurement was not pursued.

\subsection{NON-UNIFORM FIELDS}

The data plotted in Figure 5 are the average applied field data for both non-uniform geometries $(d=10 \mathrm{~mm})$. The average applied field has been calculated simply by dividing the peak applied voltage inducing breakdown, $V_{p k}$, by the interelectrode gap length, $d$. Higher applied voltages/fields are evidently required to initiate breakdown events using the collar-plane geometry compared to the pin-plane geometry. The difference between the average applied fields for breakdown in the two geometries is around $10 \%$. The general downward trends suggest sample conditioning with an increasing number of applied impulses for these measurements. The decreasing breakdown voltage may be a result of increasing gas content in the oil due to previous breakdown events, although as already mentioned in section 1 , the study involved testing the oil "as received", and dissolved gas or water content was not analyzed.

As in the over-voltage measurements, the time to breakdown was generally longer for the collar configuration as compared with the pin geometry. The longer times to breakdown for the collar translate to slower streamer propagation, as summarised in Table 5. Comparing the propagation velocities to those for gaps subjected to over-voltages with $100 \mathrm{~ns}$ rise-time
(Table 2), the values are similar for collar-plane geometry. The velocities for pin-plane geometry however, are substantially faster for the over-voltage measurements compared to the velocities obtained from breakdown-initiation measurements.

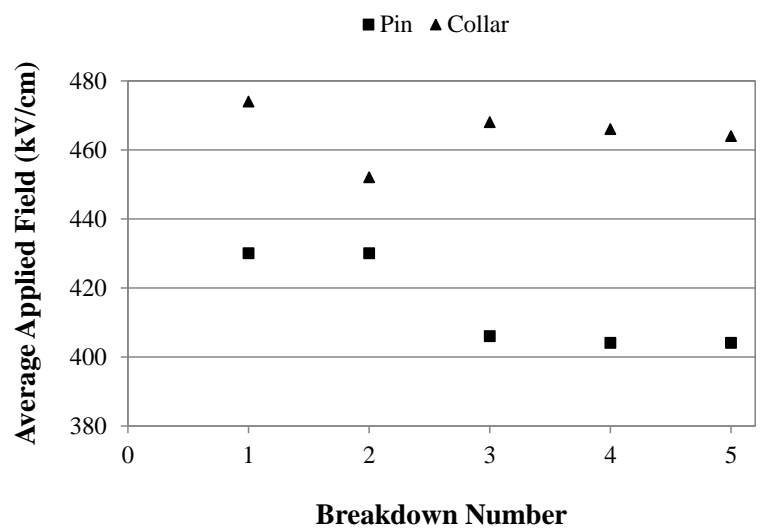

Figure 5. Average applied field $\left(V_{p k} / d\right)$ versus breakdown number (5 individual breakdowns) for breakdown-initiation measurements under nonuniform geometries.

Table 5. Implied Average Streamer Propagation Velocities for Breakdowninitiation Measurements under Non-uniform Field Geometries.

(5 Individual Breakdowns).

\begin{tabular}{c|c|c|c|c|c}
\hline \multirow{2}{*}{ Electrodes } & \multicolumn{5}{|c}{ Average Streamer } \\
\cline { 2 - 6 } & 1 & 2 & 3 & 4 & 5 \\
\hline Pin-plane & 28 & 18 & 27 & 19 & 21 \\
\hline Collar-plane & 17 & 21 & 14 & 15 & 17 \\
\hline
\end{tabular}

\subsection{UNIFORM FIELDS}

For a 4-mm open oil gap between plane-parallel electrodes, the highest applied field required to initiate breakdown was $765 \mathrm{kV} / \mathrm{cm}$, as measured for the final breakdown event $\left(V_{p k}=\right.$ $306 \mathrm{kV}$ ). The peak applied voltage inducing breakdown, $V_{p k}$, is listed in Table 6 , along with the voltage at breakdown, $V_{b r}$, and the time to breakdown, $t_{b r}$. The applied field for the other breakdown events was in the range $515 \mathrm{kV} / \mathrm{cm}$ to $670 \mathrm{kV} / \mathrm{cm}$. The average breakdown field (calculated by dividing the breakdown voltage, $V_{b r}$, by the inter-electrode gap length) varied form $405 \mathrm{kV} / \mathrm{cm}$ up to $750 \mathrm{kV} / \mathrm{cm}$. As expected, the average applied fields necessary to cause breakdown are higher than those obtained in non-uniform geometries.

Table 6. Peak Applied Voltage, $V_{p k}$, Voltage at Breakdown, $V_{b r}$, and Time to Breakdown, $t b r$, for Breakdown-initiation Measurements with Plane-parallel Electrodes (5 Individual Breakdowns).

\begin{tabular}{c|c|c|c|c}
\hline $\begin{array}{c}\text { Rise-time } \\
\text { (inter-electrode gap) }\end{array}$ & & $V_{p k}(\mathrm{kV})$ & $V_{b r}(\mathrm{kV})$ & $\operatorname{tbr}(\mathrm{ns})$ \\
\hline \multirow{4}{*}{$\begin{array}{c}100 \mathrm{~ns} \\
(d=4 \mathrm{~mm})\end{array}$} & 1 & 226 & 197 & 376 \\
\cline { 2 - 5 } & 2 & 268 & 232 & 344 \\
\cline { 2 - 5 } & 3 & 229 & 197 & 368 \\
\cline { 2 - 5 } & 4 & 206 & 162 & 504 \\
\cline { 2 - 5 } & 5 & 306 & 300 & 240 \\
\hline
\end{tabular}

As might be expected, the higher the applied field required for breakdown initiation, the shorter the delay time until 
breakdown on the falling edge of the impulse. The corresponding implied streamer velocities are displayed in Table 7. The calculated streamer velocities are generally slower than those in non-uniform fields, by up to 2.5 times in collar-plane geometry, and by a maximum of 3.5 times in pinplane geometry.

Table 7. Implied Average Streamer Propagation Velocities for Breakdowninitiation Measurements with Plane-parallel electrodes. (5 Individual Breakdowns).

\begin{tabular}{c|c|c|c|c|c}
\hline \multirow{2}{*}{ Electrodes } & \multicolumn{5}{|c}{ Average Streamer } \\
& \multicolumn{4}{|c}{ Propagation Velocity $(\mathrm{km} / \mathrm{s})$} \\
\cline { 2 - 6 } & 1 & 2 & 3 & 4 & 5 \\
\hline Plane-parallel & 11 & 12 & 11 & 8 & 17 \\
\hline
\end{tabular}

\section{DISCUSSION}

The breakdown characteristics of open oil gaps have been studied under three different electrode geometries, and with two applied voltage wave-shapes.

In oil gaps subjected to over-voltages, the time to breakdown under collar-plane geometry was longer than that under pin-plane geometry for rise-times of both $100 \mathrm{~ns}$ and $1 \mu \mathrm{s}$. As would be expected, the pre-breakdown delay times were longer for the $1-\mu$ s rise-time for each of these nonuniform geometries.

Despite the shorter breakdown times for the pin electrode under $1-\mu$ s rise-time, the breakdown voltages in Table 1 are very similar to those for the collar electrode. This is facilitated by a period of approximately $400 \mathrm{~ns}$ around the impulse peak where the rate of voltage change is low.

The times to breakdown are generally much longer for collar-plane geometry than pin-plane geometry, suggesting that the field enhancement provided by the pin is playing a greater role compared with that for the collar.

Only the average electric fields have been discussed thus far, calculated as the ratio of the breakdown voltage and the inter-electrode gap length. In order to estimate the peak field for the non-uniform geometries, these electrode arrangements were modelled using the electrostatic field solver QuickField (Tera Analysis Ltd.). The solver is based on finite element analysis, and produces a rotationally symmetric solution. The applied voltage was $400 \mathrm{kV}$ in all cases. When the pin-plane geometry was modelled using QuickField, the estimated peak fields were $\sim 2.8 \mathrm{MV} / \mathrm{cm}$ for $d=8.5 \mathrm{~mm}$ and $\sim 2.5 \mathrm{MV} / \mathrm{cm}$ for $d=10 \mathrm{~mm}$. This results in a calculated field enhancement factor (defined here as the maximum field in the gap divided by the average field in the gap) in the range 5.95-6.25 for the pin-plane geometry. The estimated peak field for the collarplane geometry was $\sim 1.5 \mathrm{MV} / \mathrm{cm}$ for $d=8.5 \mathrm{~mm}$, and $\sim 1.3 \mathrm{MV} / \mathrm{cm}$ for $d=10 \mathrm{~mm}$, yielding a field enhancement factor in the range 3.19-3.25.

In analysing hemispherically-capped rod-plane gaps, Qiu [20] states that the field non-uniformity factor, $f$, can be estimated using the phenomenological equation (1):

$$
f=0.45 \frac{d}{r_{p}} \frac{\ln \left(6 d / r_{p}\right)}{\ln \left(d / r_{p}\right)}
$$

when the condition $3<d / r_{p}<500$ is satisfied. The values of $d / r_{p}$ for the non-uniform geometries investigated in the present study are within this range, and analysis of $f$ using equation (1) yields values of $\sim 14.4\left(d=10 \mathrm{~mm} ; d / r_{p}=20\right)$ and $\sim 12.5(d=$ $\left.8.5 \mathrm{~mm} ; d / r_{p}=17\right)$. According to Torshin [14], "the leader process forms directly from the electrode at the voltage corresponding to a breakdown probability (1 to 10\%)" for such values of $f$ (from 10 to 15). Torshin states that average field strength of $350-400 \mathrm{kV} / \mathrm{cm}$ within a critical volume of oil $\left(\sim 0.04 \mathrm{~cm}^{3}\right)$ in the vicinity of the high-voltage electrode is required for leader formation, and that "weak luminous brushtype channels" develop at speeds of $1.5-40 \mathrm{~km} / \mathrm{s}$ in the first instance.

In the present study, the pre-breakdown delay times associated with open oil gaps were longer for $1 \mu \mathrm{s}$ rise-time than for $100 \mathrm{~ns}$ rise-time, with fixed inter-electrode gap. The time-to-breakdown data has been used to calculate minimum implied average streamer velocity. The velocity of sound in mineral oil has previously been measured as $1.5 \mathrm{~km} / \mathrm{s}$ [21], so streamers generated under the conditions investigated here propagate with supersonic velocity in all cases. The maximum calculated velocity was around $40 \mathrm{~km} / \mathrm{s}$ under pin-plane geometry with rise-time $100 \mathrm{~ns}$ (average field $\sim 470 \mathrm{kV} / \mathrm{cm} ; f$ 12.5), corresponding with the observations of Torshin [14] on leaders described above. Propagation velocities for collarplane geometry were similar for both over-voltage and breakdown-initiation measurements, but under pin-plane geometry the velocities were faster when the gap was subjected to over-voltage tests (maximum of $43 \mathrm{~km} / \mathrm{s}$ ) rather than breakdown-initiation tests (minimum of $18 \mathrm{~km} / \mathrm{s}$ ), due to the higher peak voltage. For all geometries, the ratio of propagation velocity is around 2.5-3:1 for 100-ns rise-time impulses ( $d V / d t$ up to $4 \mathrm{MV} / \mu \mathrm{s}$ ) compared to $1 \mu \mathrm{s}$ rise-time impulses ( $d V / d t$ up to $350 \mathrm{kV} / \mu \mathrm{s})$, a direct effect of the shorter times to breakdown discussed above.

Under plane-parallel electrodes, which were used to provide uniform field distribution, the highest average field required to cause breakdown was $765 \mathrm{kV} / \mathrm{cm}$. This field was recorded twice for breakdown of a 4-mm oil gap: first in over-voltage tests with a $1-\mu$ s rise-time, and also in breakdown-initiation measurements with rise-time $100 \mathrm{~ns}$.

Comparing the results obtained in breakdown-initiation tests to those previously obtained in liquid-solid gaps for the same type of test [6], the applied field required to initiate breakdown was always lower with a spacer between the electrodes, for both non-uniform geometries. The minimum average applied field $\left(V_{p k} / d\right)$ inducing breakdown in an open oil gap was $\sim 405 \mathrm{kV} / \mathrm{cm}$ (pin-plane electrodes), and the maximum field was $\sim 390 \mathrm{kV} / \mathrm{cm}$ with the inclusion of a polypropylene surface (collar-plane electrodes). In all cases, higher fields were required to initiate breakdown in collar-plane geometry.

The times to breakdown can also be compared with those previously obtained in composite liquid-solid gaps for the same voltage wave-shape, and associated with the breakdown voltages reported in [5]. For the $1 / 6.5 \mu \mathrm{s}$ wave-shape, the baseline times to breakdown of the oil were found to be around $\sim 750 \mathrm{~ns}$ and $\sim 1250 \mathrm{~ns}$ for the pin-plane and collar-plane geometries, respectively (see Figure 4). In terms of surface 
breakdown, the closest values to these baseline levels were $\sim 680 \mathrm{~ns}$ in pin-plane geometry, and $\sim 715 \mathrm{~ns}$ in collar-plane geometry, both with low-density polyethylene $\left(\begin{array}{ll}\varepsilon_{r} & \sim 2.2\end{array}\right)$ surfaces. Taking the lower extreme case as an example, the average times to breakdown for Torlon $\left(\varepsilon_{r} \sim 3.9\right)$ were $\sim 225 \mathrm{~ns}$ for the pin and $\sim 250 \mathrm{~ns}$ for the collar. The corresponding reductions in breakdown time are 3.3 times and 5 times, respectively, when compared to the baseline times for the open oil gap stated above. It should be noted that the calculated standard deviation was always much lower for the open oil gap, suggesting a more consistent breakdown mechanism. It may be the case that the presence of a solid surface acts to promote the conditions required for the initiation of leader discharges (or fast streamers), resulting in shorter times to breakdown. This is facilitated by enhanced field in the direction of streamer propagation at the liquid-solid interface, and by restriction of branching of the streamer by the physical presence of the solid [22].

When considering the $100 / 600 \mathrm{~ns}$ wave-shape, the introduction of a solid sample between the electrodes again causes a reduction in the delay time to breakdown. For pinplane geometry, the baseline pre-breakdown delay time in oil was found to be $\sim 225 \mathrm{~ns}$ (see Figure 2). The implied average streamer propagation velocity, calculated by dividing the interelectrode gap length by the time to breakdown, is $38 \mathrm{~km} / \mathrm{s}$. Upon comparing this with the data in [6], it becomes clear that the introduction of a solid spacer between the electrodes generally causes a reduction in this delay time, by $\sim 1.5-2.5$ times. This results in implied streamer propagation velocity of greater than $100 \mathrm{~km} / \mathrm{s}$ in some cases. Generally, the closer the relative permittivity, $\varepsilon_{r}$, of the solid to that of the surrounding mineral oil $\left(\varepsilon_{r} \sim 2.2\right)$, the longer the pre-breakdown time delay, and polypropylene $\left(\varepsilon_{r} \sim 2.25\right)$ and low-density polyethylene $\left(\varepsilon_{r}\right.$ 2.2) offered the best overall performance. In collar-plane geometry in oil, the baseline pre-breakdown delay time was more than double that for pin-plane geometry, at 500 ns (see Figure 2), corresponding with an average streamer propagation velocity of $17 \mathrm{~km} / \mathrm{s}$. In this case, the effect of including a solid spacer between the electrodes is much more pronounced, with a decrease in the pre-breakdown delay time by a factor of five in most cases [6].

The assumption in calculating streamer velocity using information only on the time to breakdown and the interelectrode gap length is that the statistical time lag will be short at such high electric field and rate of voltage rise, and therefore the major contributor to the time to breakdown will be the formative time lag associated with streamer development and propagation. As discussed in Section 3.1, spatial and temporal imaging of streamer development would be required in order to directly investigate streamer velocity.

In future work, von Laue plots of breakdown time distributions may help to separate the contributions of statistical time lag and formative time lag to the overall time to breakdown, for both liquid breakdown, and interfacial liquidsolid breakdown.

\section{CONCLUSIONS}

Considering the results on liquid breakdown described herein and the parallel liquid-solid breakdown results reported in [3-6] as a whole, the following general conclusions can be drawn, for breakdown times of the order of hundreds of ns:

- higher applied fields are required to initiate the breakdown process in an open oil gap compared to the case when a solid spacer is present;

- the times to breakdown are longer for open oil gaps compared to oil gaps with solid spacers, showing that the presence of the solid surface acts to decrease pre-breakdown delay times; and

- based upon typical propagation velocities described in [1518], shorter time to breakdown with fixed inter-electrode gap length suggests propagation of streamers (as used to describe all propagating pre-breakdown structures) in faster modes for faster rate of voltage rise. Furthermore, the times to breakdown reported here are consistent with the propagation of leader discharges, as discussed in [14].

\section{ACKNOWLEDGMENT}

M.P.W. gratefully acknowledges financial support of the study from AWE Aldermaston.

\section{REFERENCES}

[1] T. H. Martin, A. H. Guenther, and M. Kristiansen, Eds., J. C. Martin on Pulsed Power, Plenum Press, New York and London, 1996.

[2] G. A. Mesyats, Pulsed Power, Springer: New York, 2005.

[3] M. P. Wilson, M. J. Given, I. V. Timoshkin, S. J. MacGregor, T. Wang, M. A. Sinclair, K. J. Thomas, and J. M. Lehr, "Impulse-driven surface breakdown data: A Weibull statistical analysis", IEEE Trans. Plasma Sci., accepted for publication, 2012.

[4] M. P. Wilson, I. V. Timoshkin, M. J. Given, S. J. MacGregor, M. A. Sinclair, K. J. Thomas, and J. M. Lehr, "Effect of applied field and rate of voltage rise on surface breakdown of oil-immersed polymers", IEEE Trans. Dielectr. Electr. Insul., Vol. 18, pp. 1003-1010, 2011.

[5] M. P. Wilson, M. J. Given, I. V. Timoshkin, S. J. MacGregor, M. A. Sinclair, K. J. Thomas, and J. M. Lehr, "Impulse-breakdown characteristics of polymers immersed in insulating oil", IEEE Trans. Plasma Sci., Vol. 38, pp. 2611-2619, 2010.

[6] M. P. Wilson, S. J. MacGregor, M. J. Given, I. V. Timoshkin, M. A. Sinclair, K. J. Thomas, and J. M. Lehr, "Surface flashover of oilimmersed dielectric materials in uniform and non-uniform fields", IEEE Trans. Dielectr. Electr. Insul., Vol. 16, pp. 1028-1036, 2009.

[7] M.P. Wilson, I.V. Timoshkin, M.J. Given, S.J. MacGregor, M.A. Sinclair, K.J. Thomas, and J.M. Lehr, "Effect of electrode geometry and rate of voltage rise on streamer propagation in mineral oil", IEEE Int'l. Conf. Dielectr. Liq., ICDL, 2011.

[8] J. Mankowski, J. Dickens, and M. Kristiansen, "High voltage subnanosecond breakdown”, IEEE Trans. Plasma Sci., Vol. 26, pp. 874881, 1998.

[9] M. Krins, H. Borsi, and E. Gockenbach, "Impact of carbon particles on the impulse flashover behaviour of different solid/liquid interfaces in a non-uniform field", Int'l. Sympos. Electr. Insul. Materials, pp. 363-368, 1998.

[10] R. Bartnikas, "Electrical Insulating Liquids," Engineering Dielectrics, Philadelphia, USA, Vol. 3, pp. 284-301, 1994.

[11] C. Mazzetti, M. Pompili, and E. O. Forster, "Study of the time to breakdown in transformer oil under impulse conditions", IEEE Trans. Electr. Insul., Vol. 25, pp. 1111-1116, 1990.

[12] L. Lundgaard, D. Linhjell, B. Gunnar, and S. Svein, "Propagation of positive and negative streamers in oil with and without pressboard interfaces", IEEE Trans. Dielectr. Electr. Insul., Vol. 5, pp. 388-395, 1998. 
[13] O. Lesaint, “'Streamers” in liquids: relation with practical high voltage insulation and testing of liquids", IEEE Int'l. Conf. Dielectr. Liquids (ICDL), pp 1-6, 2008.

[14] Y. V. Torshin, "On the existence of leader discharges in mineral oil", IEEE Trans. Dielectr. Electr. Insul., Vol. 2, pp. 167-179, 1995.

[15] R. Liu, C. Törnkvist, V. Chandramouli, O. Girlanda, and L. A. A. Pettersson, "Ester fluids as alternative for mineral oil: The difference in streamer velocity and LI breakdown voltage", IEEE Conf. Electr. Insul. (CEIDP), pp. 543-548, 2009.

[16] R. Badent, K. Kist, and A. J. Schwab, "Streamer initiation and propagation in insulating oil in weakly non-uniform fields under impulse conditions", IEEE Int'l. Sympos. Electr. Insul., Vol. 2, pp. 720723, 1996.

[17] G. Massala, and O. Lesaint, "A comparison of negative and positive streamers in mineral oil at large gaps", J. Phys. D: Appl. Phys., Vol. 34, pp. 1525-1532, 2001.

[18] C. T. Duy, O. Lesaint, A. Denat, and N. Bonifaci, "Streamer propagation and breakdown in natural ester at high voltage", IEEE Trans. Dielectr. Electr. Insul., Vol. 16, pp. 1582-1594, 2009.

[19] E. O. Forster, and P. Wong, "High speed laser Schlieren studies of electrical breakdown in liquid hydrocarbons", IEEE Trans. Electr. Insul., Vol. 12, pp. 435-442, 1977.

[20] Y. Qiu, "Simple expression of field nonuniformity factor for hemispherically capped rod-plane gaps", IEEE Trans. Electr. Insul., Vol. 21, pp. 673-675, 1986.

[21] R. Ohyama, A. Watson, and J. S. Chang, "Electrical current conduction and electrohydrodynamically induced fluid flow in an AW type EHD pump", J. Electrostatics, Vol. 53, pp. 147-158, 2001.

[22] O. Lesaint, and G. Massala, "Transition to fast streamers in mineral oil in the presence of insulating solids", IEEE Int'l. Sympos. Electr. Insul., Vol. 2, pp. 737-740, 1996.

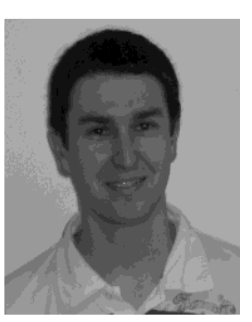

Mark P. Wilson (M'10) was born in Stranraer, Scotland, in 1982. He received the B.Eng. (with honours), M.Phil., and Ph.D. degrees in electronic and electrical engineering from the University of Strathclyde, Glasgow, U.K., in 2004, 2007, and 2011, respectively. He is presently working as a Teaching Associate at the University of Strathclyde, where he continues to investigate surface flashover of solids immersed in insulating oil. Mark is a member of the IEEE Dielectrics and Electrical Insulation Society, the IEEE Nuclear and Plasma Sciences Society, from whom he received a Graduate Scholarship Award in 2011, and the IET.

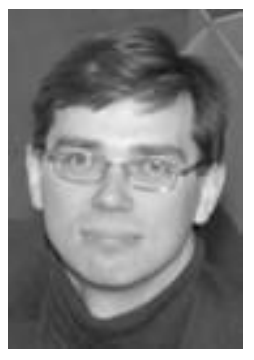

Igor V. Timoshkin (M'07) received the degree in physics from the Moscow State University (Russia) in 1992, and the Diploma and the Ph.D. degree from the Imperial College of Science, Technology and Medicine, (London, UK) in 2001. After graduation from MSU he worked as a Researcher at Moscow State Agro-Engineering University, and then at the Institute for High Temperatures of Russian Academy of Sciences before moving to ICSTM in 1997. He joined the Department of Electronic and Electrical Engineering of the University of Strathclyde (Glasgow, UK) in 2001 as an Academic Visitor, where he became a Research Fellow in 2002, a Lecturer in 2006, and a Senior Lecturer in 2011. His research interests include properties of solid and liquid dielectric materials, electronics of plasma discharges in condensed media, practical applications of electro-hydraulic and high-power ultrasound pulses, bio-dielectrics and effects of electromagnetic fields on biological objects.

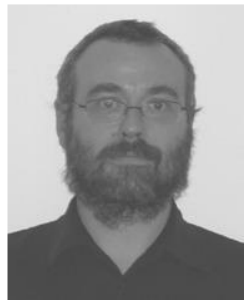

Martin J. Given (M'99-SM'11) is currently a Senior Lecturer in the Department of Electronic and Electrical Engineering at the University of Strathclyde. He received a degree in physics from the University of Sussex in 1981 and a PhD in electronic and electrical engineering from the University of Strathclyde in 1996. His research interests include, ageing processes and condition monitoring in solid and liquid insulation systems, high speed switching and pulse power applications.

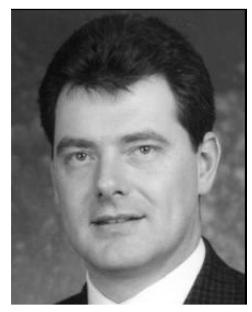

Scott J. MacGregor (M'95) received the B.Sc. and $\mathrm{Ph} . \mathrm{D}$. degrees from the University of Strathclyde, Glasgow, U.K., in 1982 and 1986, respectively. He is currently with the University of Strathclyde, where he became a Pulsed-Power Research Fellow in 1986, a Lecturer in pulsed-power technology in 1989, a Senior Lecturer in 1994, and a Reader and a Professor of high-voltage engineering in 1999 and 2001, respectively. He has been Dean of Engineering at the University of Strathclyde since January 2010. His research interests include high-voltage pulse generation, high-frequency diagnostics, high-power repetitive switching, high-speed switching, electronic methods for food pasteurization and sterilization, generation of high-power ultrasound (HPU), plasma channel drilling, pulsedplasma cleaning of pipes, and stimulation of oil wells with HPU.

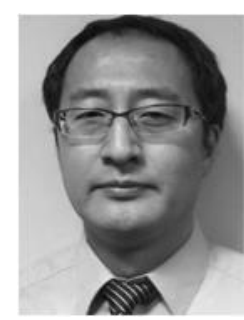

Tao Wang received the B.Eng and M.Sc degrees from Northeast China Dianli University (China) in 1993 and 1996 respectively, and the Ph.D. degree from the University of Strathclyde (Glasgow, UK) in 2005. He then joined the Newland Entech as a research fellow developing high efficiency industrial ozone generator. He joined the department of Electronic and Electrical Engineering of University of Strathclyde as a lecturer in 2010. His research interests include non-thermal gas discharges and their applications in gas synthesis, water disinfection and advanced oxidation process in water.

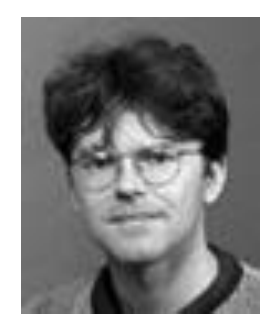

Mark Sinclair (M'03) received the B.Sc. degree from the Victoria University of Manchester, UK, in 1990 and then went to St. Andrews and Strathclyde Universities in Scotland to receive an M.Sc. degree in Laser Engineering and Pulsed Power Technology. In 1992 he joined the Pulsed Power Group at AWE. Initially he worked on the E Minor machine moving on to Mogul E which was optimized for radiography of dense objects. Currently he is the technical lead and Team Leader for Pulsed Power at AWE running a number of programs to develop fundamental knowledge and skills, to develop new x-ray sources and to use Pulsed Power for Hydrodynamic simulation.

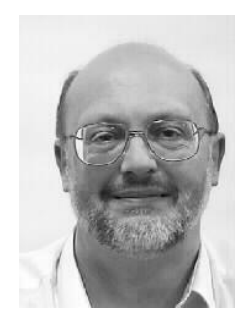

Kenneth J. Thomas (M'05-SM'10) was born in Carmarthen, South Wales in 1958. He received the B.Sc. degree in physics from Imperial College, London in 1979. He completed the Ph.D. degree in Laser Physics, also at Imperial College, in 1983 and joined the Pulsed Power Group at AWE. Since then he has been involved in the design and construction of multi-MegaVolt pulsed power machines for flash radiography and supporting research, particularly the application of Induction Voltage Adder technology to the next generation of accelerators. He is a C. Phys. and a member of the IET and IoP.

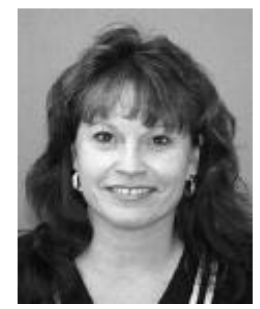

Jane Lehr (S'91-M'96-SM'02-F'08) received the B.Eng. degree from the Stevens Institute of Technology, Hoboken, NJ, and the Ph.D. degree in electro physics from Polytechnic University, New York, NY, USA. Since 2002, she has been a Member of the Technical Staff with the Exploratory Pulsed Power Technologies Branch, Sandia National Laboratories, Albuquerque, NM. Prior to joining Sandia National Laboratories, she was with the Directed Energy Directorate, Air Force Research Laboratory. Dr. Lehr is the Past President of the IEEE Nuclear and Plasma Sciences Society. 